\title{
Relationship between sleep characteristics and depressive symptoms in last trimester of pregnancy
}

\author{
Gülçin Nacar ${ }^{1}$, Sermin Timur Taşhan ${ }^{2}$
}

1. Inonu University, Faculty of Nursing / Department of Birth, Women Health and Illness, 44280 Malatya, Turkey. Fax: +904223410220 GSM: 05534770468. E-mail: gulcin.nacar@gmail.com

2. Inonu University, Faculty of Nursing / Department of Birth, Women Health and Illness, 44280 Malatya, Turkey. Fax: +90 4223410220 GSM: 05066047909 . E-mail: setimur@gmail.com

\begin{abstract}
Background: Sleep problems during pregnancy may cause many complications that reduce quality of life. Aim: This study aims to determine the relationship between pregnant women's sleep characteristics and depressive symptoms. Methods: A hospital-based, cross-sectional study was conducted. Pregnant women were selected from the population by using the an improbable random sampling method. This study sample included 436 pregnant women who met the study's inclusion criteria. To collect data, this study used an information form that was developed by the researcher after reviewing relevant literature, the Women's Health Initiative Insomnia Rating Scale (WHIIRS), and the Beck Depression Inventory (BDI). The researchers used face-to-face interviews method to collect data from the participants, pregnant women who were examined in the polyclinic. Results: This study found that $36 \%$ of participating pregnant women reported insomnia, and $38.1 \%$ experienced depressive symptoms. It also determined that participants who had problems with insomnia, who experienced a change in sleep habits, and who did not wake up feeling rested experienced depressive symptoms 1.64, 2.79, and 2.59 times more than those who didn't have these problems, respectively. who experienced a decrease in sleep, who experienced an increase in sleep, and who did not wake up feeling rested experienced depressive symptoms 1.61, 3.22, 3.53, and 2.59 times more, respectively, than those who did not have insomnia, who did not experience a change in sleep habits in pregnancy, and who woke up feeling rested, respectively. Conclusion: This study determined that there is a relationship between insomnia and depressive symptoms and that pregnant women experiencing insomnia presented with more depressive symptoms.
\end{abstract}

Keywords: Depressions, last trimester, pregnancy, sleep characteristics.

DOI: https://dx.doi.org/10.4314/ahs.v19i4.14

Cite as: Nacar G, Tashan ST. Relationship between sleep characteristics and depressive symptoms in last trimester of pregnancy. Afri Health Sci. 2019;19(4):2934-2944. https:/ / dx.doi.org/10.4314/abs.v19i4.14

\section{Introduction}

Sleep comprises about one-third of human life. It is as important as nutrition and exercise in order to have a healthy life. Therefore, sleep is one of the most essential factors that affects quality of life. Sleep has physiologi-

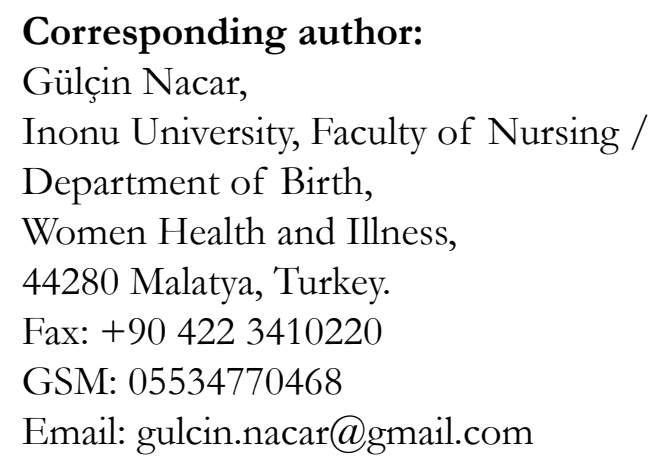

cal, psychological, and social dimensions. ${ }^{1,2}$ A vital period of life is a leading factor that affects sleep. ${ }^{3}$ For women, pregnancy is one of the most important vital periods. Although pregnancy is a natural event, it brings about many physiological, psychological, and social changes. ${ }^{4}$ Inpregnant women sleep provides a basis not only for physical and mental vitality, but also for fetal-placental growth and health. ${ }^{5}$ However, insomnia is a general health problem that women experience during pregnancy. ${ }^{6}$ The prevalence of insomnia in the pre-gestational period has been recorded as $6.1 \%{ }^{7}$ which increased to $54.3 \%$ in the first trimester, $49.3 \%$ in the second trimester, and $69.6 \%$ in the third trimester. ${ }^{8}$ According to the U.S. National Sleep Foundation data, $79 \%$ of pregnant women have experienced insomnia problems. In the first trimester, the level of insomnia is $13-80 \%$, and it increases to $66-97 \%$ in 
the second trimester. ${ }^{7}$ Studies conducted in Turkey have shown that the level of insomnia among pregnant women varies between $39 \%$ and $86 \% .^{9-11}$

The belief that pregnancy is a happy period and that it comes with emotional well-being has been abandoned; if pregnant women experience severe physical, social, and personal stress factors, events such as pregnancy, delivery, and being a parent may result in developmental and situational crises for mothers ${ }^{4}$ Depression during pregnancy is a common psychological disorder that affects both the mother's and child's health. ${ }^{12}$ The incidence of maternal depression in the pre-pregnancy period has been documented as $12.96 \% .{ }^{13}$ Furthermore, studies which have been conducted in various cultures have found that the prevalence of depression during pregnancy varies between $14.6 \%$ and $23.6 \% .^{13-15}$ Specifically, in Turkey, the prevalence of depression during pregnancy varies between $14 \%$ and $55 \% .{ }^{16-18}$

Insomnia reduces the quality of life of pregnant women and negatively affects the health of both the pregnant woman and the fetus. ${ }^{5,6,19}$ Insomnia in pregnancy may be caused by various factors ${ }^{1,6,10,13}$ and cultural differences are one of these. 8 Understanding the insomnia-depression relationship in different cultures can make it possible to develop transcultural approaches and to provide specific care. In addition, such an understanding may contribute to the development of an approach to care and treatment. A healthier pregnancy can be supportedby increasing the awareness of healthcare professionals regarding this subject. Therefore, such care can contribute to the improved health of future generations around the world. Studies in various cultures have shown that there is a relationship between sleep disorders and depressive symptoms. ${ }^{13,20,21}$ However, no study that examines the relationship of pregnant women's sleep disorders and depressive symptoms has been conducted in Turkey.

\section{General objective}

Thus, this study aimed to determine the relationship between pregnant women's sleep characteristics and depressive symptoms. This present study aimed to provide a holistic view of the sleep problems and depressive symptoms of pregnant women.

\section{Specific objectives}

This study aimed to determine the relationship between pregnant women's sleep characteristics and depressive symptoms. To do so, two objectives were created:

1. To determine the level of depressive symptoms during pregnancy; and.

2. To determine sleep characteristics during pregnancy.

To determine sleep characteristics that are risk factors for depressive symptoms during pregnancy.

\section{Method}

This was a cross-sectional study. The study was conducted in the obstetrics and gynaecology polyclinics of a public hospital located in the East of Turkey between December $1^{\text {st }}, 2014$ and March 30 ${ }^{\text {th }}, 2015$. The study population included pregnant women seeking prenatal care at the obstetrics and gynaecology outpatient clinic within the dates of the study.

\section{Study sample}

The sample consisted of 436 pregnant women. A power analysis was computed to determine the adequacy of the power for the study. With a sample size of 436, at a level of significance of 0.05 , a power of 0.95 , a $95 \%$ confidence interval was determined for this study. Using an improbable random sampling method, the researcher continued to include pregnant women from the population who met the study inclusion criteria until the sampling number was achieved. The inclusion critria of this study were as follows: being between 18 and 35 years-of-age; expecting a single birth; being 28 or more weeks pregnant; not suffering from any risk factor during pregnancy (e.g., preeclampsia, gestational diabetes, intrauterine growth retardation); not having any systemic disease (e.g., diabetes, hypertension, cardiovascular disorders); and not suffering from a psychiatric disease (e.g., depression) presently or before becoming pregnant. The exclusion criteria of this study included communication problems, which some pregnant womendemonstrated, and a desire to withdraw from the research.

\section{Data collection}

Using face-to-face interviews, data were collected from the pregnant women who met all of the study inclusion criteria and who were examined in the polyclinic. The interviews were held on weekdays (Monday, Wednesday, and Friday). During the data collection period of the study, the Participant Description Form, described below, was used. Additionally, the Women's Health Initiative Insomnia Rating Scale (WHIIRS) and the Beck Depression Inventory (BDI) were also used.

African Health Sciences Vol 19 Issue 4, December, 2019 
Information Form: This form was developed by the researcher after reviewing the literature. It included a total of 15 questions. Of the questions, four were used to determine the pregnant women's sociodemographic characteristics (age, educational level, income level, occupation), two were used to determine their obstetrical characteristics (pregnancy number, pregnancy week), and nine were used to determine their sleep habits (average amount of sleep, the difference between pre-pregnancy and current sleep habits, discomfortcausing insomnia problems, snoring habits, whether they had taken measures to reduce insomnia, and what measures were taken). . $^{3,19,22}$ Whether subjects had psychiatric diagnoses or not was determined according to their own statements. The information form was piloted with 10 pregnant women to determine its ease of use and comprehensibility. There was no change in the form after the pre-implementation process.

Women's Health Initiative Insomnia Rating Scale (WHIIRS): This scale was developed by Levine et al. (2003); Timur and Şahin (2009) performed its validity and reliability analyses for Turkey WHIIRS is a 5-item Likerttype scale. The first four questions in the WHIIRS aim to identify the beginning of insomnia, sleep-maintenance insomnia, and the state of waking up early in the mornings. The last question is associated with sleep quality. Participants should respond to each question according to their experiences by considering the frequency in each week during the four weeks prior. Each question in the WHIIRS is scored between 0 and 4 and evaluated according to the answers. A score of 0 , attained after answering the first four questions, shows no problem with insomnia while a score of 4 indicates that insomnia is experienced at least five times perweek. The highest possible score of the scale indicates the highest incidence of insomnia symptoms. The minimum possible score of the scale is 0 , and the maximum is 20 . Ten or more points attained on the WHIIRS indicates that there is a problem with insomnia. ${ }^{23}$ A study conducted by Timur and Şahin (2009) found the Cronbach's alpha value to be 0.85 .

The original version of the scale was developed by Beck et al. (1961).. The BDI does not rank the etiology of diseases; it provides information on the depressive symptoms and attitudes related to an individual's' emotional, somatic, and motivational states. The BDI form has four choices for each of the 21 categories of symptoms that is covers. This form asks patients to select the most appropriate sentence in order to express how they have felt during the prior seven days, including the day the form was administered. Scores on each item can range from 0 to 3. The highest possible score is 63 . Higher totals indicate more severe depression or a higher level of depressive symptoms. Furthermore, the aim of the BDI scale is not to diagnose depression but to provide an objective determination of the severity of depressive symptoms. The scale has no sub-dimensions. For persons who are 15 years-of-age or older, the validity and reliability analyses of the BDI were performed by Tegin and Hisli. Tegin conducted a study with 40 university students and 30 depressive patients and determined that the BDI was appropriate for the Turkish language and was reliable for both groups. While the Turkish version of the BDI was being developed, cut-off points were examined, and a score of 17 was decided upon. The reliability coefficient of the scale for students was found to be 0.65 using the test-retest method. However, using the two-half method, the reliability coefficients were determined to be 0.78 and 0.61 for students and for depressive patients, respectively. 3,24

\section{Ethical considerations}

Before initiating the study, the researcher received written permission from the ethical committee (2014/157) and from the hospital where this study was conducted. The purpose and the procedures to be performed were explained to the pregnant women. These women were told that they were free to withdraw at any time they wanted. Those who agreed to take part were included. After the data collection, questions about the pregnancy and reproductive health of pregnant women were answered by the researcher.

\section{Statistical analysis}

The researcher performed the data analysis in a computer environment using the SPSS 15.0 software. For statistical evaluation, this study used percentage distributions, arithmetic means, standard deviations, the chi-square test, Spearman's correlation test, Cronbach's alpha reliability analysis, and the Backward Stepwise Logistic Regression analysis test. Results were evaluated using a 95\% confidence interval and a significance level of $\mathrm{p}<0.05$. 


\section{Results}

The mean age of the pregnant women who participated in the study was $27.7 \pm 5.0$ years (min: 18; max: 35), and $88.8 \%$ of participants identified as housewives. Also, $36.9 \%$ of the women were literate or had graduated from primary school. It was determined that $59.2 \%$ of the women had an income that was less than their expenses. The mean total pregnancy number of the participants was $2.3 \pm 1.3$, and it was determined that $30.7 \%$ were experiencing their first pregnancy while $52.6 \%$ were pregnant for the second or third time. According to study findings, the mean pregnancy week of the participants was $36.4 \pm 2.6$.

Insomnia in the sample was determined using the Women's Health Initiative Insomnia Rating Scale $(\geq 10)$. The distribution of pregnant women according to their sleep characteristics and depressive symptoms is shown in Table 1 . This study found that $36 \%$ of the participating pregnant women had insomnia. Additionally, Tthis study found the Cronbach's alpha value of the WHIIRS to be 0.64 . While the participants' daily average amount of sleep was determined to be $6.4 \pm 2.4,62.2 \%$ of the participants slept each day for seven or fewer hours. A majority of the pregnant women $(86.7 \%)$ reported that they had experienced a change in their sleep habits. Of the pregnant women who experienced a change in their sleep habits, $80.5 \%$ stated that they had slept more before becoming pregnant. This study showed that of the participating pregnant women, $17.7 \%$ snored, $25.7 \%$ slept during the day, and $61.5 \%$ felt they were not rested when they woke up in the morning (Table 1).

Table 1: The distribution of pregnant women's sleep characteristics and depressive symptoms

\begin{tabular}{|c|c|c|c|}
\hline \multirow{2}{*}{$\begin{array}{l}\text { Sleep characteristics } \\
\text { Insomnia }\end{array}$} & \multirow[t]{2}{*}{$\mathbf{N}$} & \multicolumn{2}{|l|}{$\%$} \\
\hline & & & $T$ \\
\hline Yes & 157 & 36.0 & \\
\hline No & 279 & 64.0 & \\
\hline Sleep duration /day* & & & | \\
\hline$\leq 7$ & 271 & 62.2 & \\
\hline$>7$ & 165 & 37.8 & \\
\hline \multicolumn{4}{|l|}{ Change in sleep habits in pregnancy } \\
\hline Yes & 378 & 86.7 & \\
\hline No & 58 & 13.3 & \\
\hline \multicolumn{4}{|l|}{ Type of change in sleep habits ( $n: 378)$} \\
\hline More before pregnancy-less now & 351 & 80.5 & \\
\hline Less before pregnancy-more now & 27 & 6.2 & \\
\hline \multicolumn{4}{|l|}{ Change in sleep habits in pregnancy } \\
\hline Decrease in sleep & 351 & 80.5 & \\
\hline Increase in sleep & 27 & 6.2 & \\
\hline No change & 58 & 13.3 & \\
\hline \multicolumn{4}{|l|}{ Snoring } \\
\hline Yes & 77 & 17.7 & \\
\hline No & 359 & 82.3 & \\
\hline \multicolumn{4}{|l|}{ Sleep during the day } \\
\hline Yes & 112 & 25.7 & \\
\hline & 324 & 74.3 & \\
\hline \multicolumn{4}{|l|}{ Waking up feeling rested in the morning } \\
\hline Yes & 168 & 38.5 & \\
\hline No & 268 & 61.5 & \\
\hline \multicolumn{4}{|l|}{ Causes of insomnia ** } \\
\hline Pain (Back, leg, pubic, contraction ) & 260 & 59.6 & \\
\hline Frequent going to toilet at night & 239 & 54.8 & \\
\hline Not finding a comfortable position in bed & 153 & 35.1 & \\
\hline Twitching and spasms in legs & 48 & 11.0 & \\
\hline Psychological problems & 31 & 7.1 & \\
\hline Not breathing comfortably & 9 & 2.1 & \\
\hline Heartburn & 9 & 2.1 & \\
\hline Drugs used & 2 & 0.5 & \\
\hline Other ${ }^{8}$ & 2 & 0.5 & \\
\hline Taking preventive actions to reduce insomnia & I & | & \\
\hline Yes & 127 & 41.5 & \\
\hline No & 179 & 58.5 & \\
\hline Preventive actions*** & 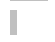 & 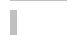 & \\
\hline Consuming milk and dairy products & 82 & 56.2 & \\
\hline Taking a shower & 48 & 32.9 & \\
\hline Drug use & 6 & 4.1 & \\
\hline Walking & 4 & 2.8 & \\
\hline Massage & 3 & 2.1 & \\
\hline Other $^{*}$ & 3 & 2.1 & \\
\hline Depressive symptoms ${ }^{t}$ & I & 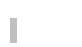 & \\
\hline Yes & 166 & 38.1 & \\
\hline No & 270 & 61.9 & \\
\hline
\end{tabular}


In this study, the participants reported that they suffered from insomnia because they felt pain (e.g., back, leg, pubic, contraction) $(59.6 \%)$, used the toilet frequently at night $(54.8 \%)$, were unable to find a comfortable position in bed (35.1\%), and had twitching and spasms in their legs $(11 \%)$. Of the participants, $41.5 \%$ stated that they took preventive actions to reduce insomnia. The most common preventive actions against insomnia were consuming milk and dairy products and taking a shower (Table, 1). The presence of depressive symptoms in the pregnant women was determined using the BDI $(\geq 17)$. This study found the Cronbach's alpha level of the BDI to be 0.78. This study Furthermore, it was also found that $38.1 \%$ of participating pregnant women had experienced depressive symptoms. The BDI mean score was $15.27 \pm 6.45$ (Table 1).

The comparison between the pregnant women who had depressive symptoms and their sleep habits is shown Table 2. had ). (Table, 2).

According to these results, pregnant women who experienced insomnia also, had a decline in sleeping duration during pregnancy, did not wake up feeling rested in the morning, and had more depressive symptoms $(\mathrm{p}<0.05)$. Of the pregnant women whose daily sleep time was seven or fewerhours, $70.5 \%$ experienced depressive symptoms while $29.5 \%$ of those who slept more than seven hours at night 29.5\% had depressive symptoms ( $\mathrm{p}<$ 0.05) (Table 2).

This study found that the pregnant women who woke up feeling rested in the morning $(22.9 \%)$ had fewer depressive symptoms compared to those who did not wake up feeling rested $(77.1 \%)(\mathrm{p}<0.001)$. This study did not determine a statistically significant relationship between snoring, sleeping during the day, and taking preventive actions to reduce insomnia and depressive symptoms ( $p$ $>0.05$ ) (Table 2).

Table 2: The comparison between the pregnant women having depressive symptoms and sleep habits

\begin{tabular}{|c|c|c|c|}
\hline Sleep characteristics & $\begin{array}{c}\text { Depressive } \\
\text { symptoms } \\
\text { yes }\end{array}$ & $\begin{array}{c}\text { Depressive } \\
\text { symptoms } \\
\text { no }\end{array}$ & $\begin{array}{c}\text { Test and } \\
\text { significance * }\end{array}$ \\
\hline \multicolumn{4}{|l|}{ Insomnia } \\
\hline Yes & \multirow{2}{*}{$124(44.4)$} & $155(55.6)$ & $X^{2}=13.339$ \\
\hline No & & $115(73.2)$ & $\mathrm{p}<0.001$ \\
\hline \multicolumn{4}{|l|}{ Sleep duration /day } \\
\hline$\leq 7$ & \multirow{2}{*}{$\begin{array}{c}117(70.5) \\
49(29.5)\end{array}$} & \multirow{2}{*}{$\begin{array}{l}154(57.0) \\
116(43.0)\end{array}$} & \multirow{2}{*}{$\begin{aligned} X^{2} & =7.900 \\
\mathbf{p} & <\mathbf{0 . 0 5}\end{aligned}$} \\
\hline$>7$ & & & \\
\hline \multicolumn{4}{|c|}{ Change in sleep habits in pregnancy } \\
\hline Yes & $157(94.6)$ & $221(81.9)$ & $\mathrm{X}^{2}=$ \\
\hline No & $9(5.4)$ & $49(18.1)$ & $\mathrm{p}<0$ \\
\hline \multicolumn{4}{|c|}{ Change in sleep habits in pregnancy } \\
\hline Decrease in sleep & $147(88.6)$ & $204(75.6)$ & $X^{2:} 16.741$ \\
\hline Increase in sleep & $11(6.6)$ & $16(5.9)$ & $* \mathrm{p}<0.001$ \\
\hline No change & $8(4.8)$ & $50(18.5)$ & \\
\hline \multicolumn{4}{|l|}{ Snoring } \\
\hline Yes & $33(19.9)$ & $44(16.3)$ & $X^{2}=0.908$ \\
\hline No & $133(80.1)$ & $226(83.7)$ & $p>0.05$ \\
\hline Sleep during the day & & & \\
\hline Yes & $45(27.1)$ & $67(24.8)$ & $X^{2}=0.283$ \\
\hline No & $121(72.9)$ & $203(75.2)$ & $\mathrm{p}>0.05$ \\
\hline Waking up feeling re & & & \\
\hline Yes & $38(22.9)$ & $130(48.1)$ & $X^{2}=27.686$ \\
\hline No & $128(77.1)$ & $140(51.9)$ & $\mathrm{p}<0.001$ \\
\hline Taking preventive ac & & & \\
\hline Yes & $55(40.4)$ & $72(42.4)$ & $X^{2}=0.114$ \\
\hline No & $81(59.6)$ & $98(57.6)$ & $p>0.05$ \\
\hline Total & $166(38.1)$ & $270(61.9)$ & \\
\hline
\end{tabular}


The correlations between insomnia and sleep characteristics are shown in Table 3. In this study, a weak negative correlation was found between insomnia and sleep duration (Spearman's rho $=-0.235$ ) and a change in sleep habits duringpregnancy (Spearman's rho $=-0.206$ ). A weak positive correlation was found between insomnia and waking up feeling rested in the morning (Spearman's rho $=0.231)$ (Table 3).

Table 3. Correlations between insomnia and sleep characteristics

\begin{tabular}{lc}
\hline Sleep Characteristics & Spearman's rho \\
Sleep duration/day & $-0.235^{a}$ \\
Change in sleep habits in pregnancy & $-0.206^{a}$ \\
Snoring & $0.003^{b}$ \\
Sleeping during the day & $-0.004^{b}$ \\
Waking up feeling rested in the morning & $0.231^{\mathrm{a}}$ \\
Taking preventive actions to reduce insomnia & $-0.084^{\mathrm{b}}$ \\
\hline${ }^{\mathrm{a}}$ Spearman's correlation is significant at the 0.01 level; 'Spearman's correlation is not significant at the 0.05 level.
\end{tabular}

A bivariate analysis was conducted with all variables that might be related to depression. The relationships between the depression cut-off point and insomnia, sleep duration, a change in sleep habits during pregnancy, snoring, sleeping during the day, waking up feeling rested in the morning, and taking preventive actions to reduce insomnia were assessed with the $\mathrm{X} 2$ analysis. The variables of insomnia, sleep duration, a change in sleep habits during pregnancy, and waking up feeling rested in the morning were found to be significant $(p<0.05)$. The variables found to be significant after these analyses were included in the logistic regression model. The variables that had insignificant relationships with depression, such as snor- ing, sleeping during the day, sleep duration, and taking preventive actions to reduce insomnia, were not included in the model. The results of the Backward Stepwise Logistic Regression Analysis are shown in Table 4. The results of the analysis determined that the participants who had problems with insomnia, who experienced a decrease in sleep, who experienced a an increase in sleep, and who did not wake up feeling rested also experienced depressive symptoms $1.641 .61,2.793 .22,3.53$, and 2.59 times more, respectively, than those who did not have problems with insomnia, who did not experience a change in sleep habits in pregnancy, and who woke up feeling rested, respectively (Table 4). 
Table 4. Advanced analysis of the risk factors associated with depressive symptoms in pregnant women*

\begin{tabular}{|c|c|c|c|c|c|c|c|}
\hline Risk factors for depressive symptoms & B & $\mathbf{S E}^{\mathbf{a}}$ & $\mathbf{d f}^{\mathbf{b}}$ & $\mathbf{p}$ & $\mathbf{O R}^{\mathrm{c}}$ & & $95 \% \mathrm{Cl}^{\mathrm{d}}$ \\
\hline Insomnia & I & I & I & I & I & I & I \\
\hline Yes & 0.496 & 0.229 & 1 & 0.03 & 1.64 & & $1.048-2.572$ \\
\hline Change in sleep habits in pregnancy & I & I & I & I & I & I & I \\
\hline Yes & 1.029 & 0.39 & 1 & 0.008 & 2.79 & & $1.303-6.014$ \\
\hline $\begin{array}{l}\text { Waking up feeling rested in the } \\
\text { mornin }\end{array}$ & I & I & I & I & I & I & I \\
\hline No & 0.954 & 0.229 & 1 & 0.001 & 2.59 & & $1.659-4.064$ \\
\hline \multicolumn{8}{|c|}{ *Backward Stepwise Logistic Regression SE": Standard Error; $\quad$ df $f^{b}$ : Degree of freedom; $\quad \mathrm{OR}^{c}$ : Odd's ratio; $\quad \mathrm{Cl}^{\mathrm{d}}$ : Confidence interval } \\
\hline Risk factors for depressive symptoms & $\beta$ & $\mathbf{S E}^{\mathbf{a}}$ & $\mathbf{d f}^{\mathrm{b}}$ & $\mathbf{p}$ & $\mathbf{O R}^{\mathrm{c}}$ & & $95 \% \mathrm{Cl}^{\mathrm{d}}$ \\
\hline Insomnia (referent: No) & I & I & I & I & I & I & I \\
\hline Yes & 0.476 & 0.230 & 1 & 0.03 & 1.61 & & $1.025-2.528$ \\
\hline $\begin{array}{l}\text { Change in sleep habits in } \\
\text { pregnancy (referent: No) }\end{array}$ & I & I & I & I & I & I & I \\
\hline Decrease in sleep & 1.169 & 0.40 & 1 & 0.004 & 3.22 & & $1.446-7.171$ \\
\hline Increase in sleep & 1.263 & 0.56 & 1 & 0.025 & 3.53 & & $1.174-10.658$ \\
\hline $\begin{array}{l}\text { Waking up feeling rested in the } \\
\text { morning (referent: Yes) }\end{array}$ & I & I & I & I & I & I & 更 \\
\hline No & 0.952 & 0.229 & 1 & 0.001 & 2.59 & & $1.653-4.060$ \\
\hline
\end{tabular}

\section{Discussion}

Sleep disorders may affect almost all areas of our daily life, including our mood, mental stamina, working performance, energy level, and quality of life. Sleep disorders experienced during pregnancy can be a risk factor that causes for the emergece of depressive symptoms. ${ }^{19,21}$ The fFindings of this study, which was conducted to examine the relationship between the sleep characteristics of pregnant women and depressive symptoms, were compared with the relevant literature studies.

Our This The present study found that $36 \%$ of the participating pregnant women had problems with insomnia. Facco et al. (2010) and Ko et al. (2012) also determined that $54.3 \%$ and $55.6 \%$ of pregnant women, respectively, had a problem with insomnia problem, respectively. ${ }^{23,24}$ 25, 26 Therefore, tThe rate of insomnia that was reported by the participants in the present study is was lower when compared to the study results of Facco et al. (2010) and Ko et al. (2012). Facco et al. (2010) and Ko et al. (2012) accepted set the WHIIRS cut-off score to be at 9; Hhowever, this the present study accepted set this score to be at 10 . The Thus, the percentage rate difference is likely because the cut-off scores were are different.
Moreover, Iin this research study, a negative correlation was found between insomnia, sleep duration per day, and a change in sleep habits in during pregnancy. A positive correlation was found between insomnia and waking up feeling rested in the morning. In another study carried out with pregnant women, Piinar et al. (2014) obtained results similar to our the present findings and found significant relationships between sleepig during the day, not waking up feeling rested in the morning, and finding the duration of sleep to be insufficient. ${ }^{10}$

Based on the information that sleep mediates biological and psychological restoration, ${ }^{25}$ it can be thought that deterioration of homeostasis triggers diseases. It is known that sleep disorder is a risk factor for the emergence of depression, and that depression can develop after the insomnia period. ${ }^{26}$ Based on the information that sleep the mediatestion of biological and psychological restoration by sleep, ${ }^{25,27}$ it can be considered that a deterioration of homeostasis triggers diseases. It is known that a sleep disorder may be a risk factor for the emergence of depression, and that depression may develop after experiencing insomnia. ${ }^{26,28}$ Our The present study determined that pregnant women who had problems with insomnia dis- 
played depressive symptoms 1.641 .61 times greater than those who did not have problems with insomnia (Table 34). Wolynczyk-Gmaj et al. (2017) revealed, similarly to our present research, revealed that the severity of depressive symptoms $(\mathrm{OR}=1.14, \mathrm{p}<0.001)$ were was significantly associated with insomnia during pregnancy. ${ }^{27,29}$ Additionally, Rezaei et al. (2015) conducted a study monitoring pregnant women and determined that as pregnancy progressed, the incidence of depressive symptoms in pregnant women who had insomnia also increased.20 22 A study of by Mellor et al. (2014) further found that there was a highly significant relationship between poor sleep quality and the incidence of depression.13 14 Dorheim et al. (2012) carried out In a study carried out with 2,816 pregnant women, and Dorheim et al. (2012) also identified a strong relationship between insomnia and depressive symptoms. ${ }^{12,13}$ Moreover Furthermore, in the literature, there are have been other studies showing that have shown that there is a significant relationship between sleep disorders and depressive symptoms. ${ }^{28,30,30-32}$ The findings of our study are largely in agreement with the literature.

Symptoms and physical complaints emerging during the last months of pregnancy may keep pregnant women awake more at night. ${ }^{31}$ Moreover, Ssymptoms and physical complaints emerging during the last months of pregnancy may keep pregnant women awake for longer times durations at night. ${ }^{31,33}$ While this study found the daily average amount of the pregnant women's daily average amount of sleep to be $6.4 \pm 2.4$ hours, two-thirds of the participating pregnant women participants reported that they slept seven hours or less fewer (Table 1). In the literature, there are studies showing that as the gestational age increases, sleep time decreases. ${ }^{23,24,29}$ Some pPrevious studies have also shown that sleep duration decreases as the gestational age increases. ${ }^{23,24,29,25,26,31}$ The findings of our study are largely in agreement with the literature. We found that pregnant women who slept seven or fewer hours had more depressive symptoms compared to those who slept seven or more hours; however, according to the logistic regression analysis that was completed, sleep time is not a risk factor for depressive symptoms. In the literature, there are studies showing that there is a negative relationship between sleep time and depressive symptoms. ${ }^{12,29}$ The findings of our study are largely in accord with the literature. The present study found that pregnant women who slept for seven or fewer hours had more depressive symptoms compared to those who slept for seven or more hours. HoweverAlthough, the logistic regression analysis indicated that sleep duration is was not a risk factor for depressive symptoms. Some Other studies, however, have shown a negative relationship between sleep duration and depressive symptoms. ${ }^{12,29,13,31}$

In the present our study, most of the pregnant women reported that they experienced changes in their sleep habits, and that they slept less compared to their pre-pregnancy period. e found that tThe risk for depressive symptoms was found to be 3.22 times greater among pregnant women who experienced changes a decrease in their sleep habits was 2.79 times and was also found to be 3.53 times higher among those who experienced an increase in sleep was found to be 3.53 times more higher than among compared to those who did not experience changes in sleep habits. Similarly to our study, . Dorheim et al. (2012) indicated that the risk of experiencing depression is 2.16 times higher greater for pregnant women who sleep less than 5 five hours a day than for compared to pregnant women who sleep 7-8 hours a day. However, the risk is 2.13 times higher in for pregnant women who sleep at least 10 hours a day. ${ }^{13}$ Pietikainen et al. (2018) also found that pregnant women whose sleep duration per day is was $\leq 7$ hours and or $\leq 6$ hours have had 2.23 and 3.53 times higher risks, respectively, for experiencing depression. 34 In the literature, there are studies which have shown that there is a relationship between sleep complaints and depressive symptoms. ${ }^{13,32}$ Our finding is largely in agreement with the literature.

The most serious respiratory sleep disorder is snoring., ${ }^{31}$ ${ }^{33}$ and Wwhile the frequency of snoring was found to be $4 \%$ to $-14 \%$ for non-pregnant women, this rate was reported to be $14 \%$ to $-28 \%$ during the third trimester of pregnancy. ${ }^{33,35}$ In parallel with the present study, Facco et al. (2010) determined that $16.4 \%$ of pregnant women had a snoring habit. ${ }^{23,25}$ In this the present study, about one- fifth of the pregnant women participants reported that they had a snoring habit.; tThis finding is largely in alignment with the findings of Facco et al. (2010). ${ }^{23}$, ${ }^{25}$ The present Our study did not find a significant relationship between the snoring habits of pregnant women and having depressive symptoms (Table 2). However, O'Brien et al. (2013) conducted a retrospective study on with pregnant women with who had a habitual snoring diagnosis, and found that those who had a snoring habit 
showed more depressive symptoms compared to pregnant women who did not have a snoring habit. ${ }^{18,20}$ The reason for this difference may be because O'Brien et al. (2013) conducted a their study on pregnant women with a habitual snoring habit.,1820 The present study evaluated the snoring habits of the participants by asking the women themselves if they snored. However, it is thought that questions about snoring should be directed to a person who shares a room or a bed with the participant, and the answers should be evaluated. Then, detailed research on the type of snoring should be conducted.

Sleeping during the day is an indicator that a person does not have an adequate and relaxing sleep at night.34 Sleeping during the day may be an indicator that a person does not have a sufficient and relaxing sleep at night.36 In our the present study, one fourth of the pregnant women participants reported that they slept during the day. Tsai et al. (2013) found no significant relationship between depressive symptoms and the habit of sleeping during the day,35 37 which is largely in parallel with the findings of the present study since no significant relationship was found between the habit of sleeping during the day and depressive symptoms in this study herein (Table 2).

Our Additionally, Tthe present study determined that the risk for depressive symptoms among pregnant women who woke up not feeling rested in the morning was 2.59 times more than among greater compared to those who woke up feeling rested (Table 34). Insomnia includes waking up not feeling rested, as well as complaints about having difficulty falling asleep and/or maintaining sleep. Thus, waking up not feeling rested is an indicator of insomnia and a risk factor for depressive symptoms. ${ }^{34}$ The finding of our study is mostly in accord with the literature. Insomnia may include waking up not feeling rested, as well as complaints about having difficulty falling asleep and/or maintaining sleep. ${ }^{36}$ Thus, waking up not feeling rested may be an indicator of insomnia and a risk factor for depressive symptoms.

Physical transformations such as pain, fetal moves, going to the toilet frequently at night, being unable to find a comfortable position in bed, heartburn, being unable to breathe easily, and twitching and spasms in the legs have an effect on sleep rhythm and time., ${ }^{3,22}$ Physical transformations such as pain, fetal movements, moves, going to having to use the toilet frequently at night, being unable to find a comfortable position in bed, heartburn, being unable to breathe easily, and twitching and spasms in the legs also may affect the sleep rhythm and time. ${ }^{3,12,27,13,29}$ In this study, participating pregnant women reported that they suffered from insomnia mainly becausehey felt pain, they went to used the toilet frequently at night, they could not get to a comfortable position in bed, and they had twitching and spasms in their legs, respectively (Table 1). Our finding is largely in agreement with the literature.

Our results indicate that, Moreover, about half of the, In the present study, about half of the participating pregnant women participants in the present study stated that they took preventive actions to reduce insomnia. The most common preventive actions were included consuming milk and dairy products and taking a shower, respectively (Table 1). Foods containing protein make falling asleep easier due to L-Triptofandan. Moreover, the muscle relaxant effect of hot water can make falling asleep easier. ${ }^{2}$ Foods containing protein can facilitate falling asleep sleep due to the presence of L-Triptofandan. In addition, the muscle relaxant effect of hot water can make falling asleep easier.2The coping styles of the pregnant women who participated in the current study are in alignment with the literature. However, a significant relationship between preventive actions to reduce insomnia and depressive symptoms was not found (Table 2). This may be because of the two-way relationship between insomnia and depressive symptoms. In other words, insomnia can be a primary factor that leads to depression but, it also can be a secondary complication arising as a result of depressive symptoms. ${ }^{26}$. In other words, insomnia can be a primary factor that leads to depression or a secondary complication resulting from depressive symptoms. ${ }^{28}$

It has been stated that anxiety and depression are experienced more frequently in the third trimester because of the fear of childbirth as well as, concerns about neonatal health and infant care. ${ }^{11,12}$ Studies conducted in Turkey have shown that the incidence of depression during pregnancy varies between $14 \%$ and $55 \% .{ }^{15-17,16-18}$ The present study determined that more than one third of the participating pregnant women had depressive symptoms (Table 31). The finding of our study is largely in agreement with the literature. Furthermore, studies conducted in various countries found the rate of pregnant women's depressive symptoms to be $14.6-28.4 \% .^{12-14}{ }^{13-15}$ It has been thought that this difference may arise from cultural differences, Culture has an important functions in all areas of psychi- 
atry. It has been accepted shown that social and cultural factors have a role in causing depression, as well as its symptoms and what choices are made in seeking a remedy. ${ }^{36-39}$

\section{Limitations}

This study was hospital-based, which constitutes the first limitation of the study. The second limitation of the study is the fact that the determination of whether the pregnant women had psychiatric diagnoses or not was based on their own statements. Furthermore, The low value of Cronbach's alpha of the Women's Health Initiative Insomnia Rating Scale is comprises the final limitation of the study.

\section{Conclusion}

In conclusion, pregnant women had presented with complaints about insomnia and depressive symptoms at similar levels. It was also determined that experiencing insomnia, undergoing a change in sleep habits, and waking up not feeling rested are risk factors related to depressive symptoms among pregnant women.

In the light of these results, it is recommended that healthcare staff thoroughly evaluate the complaints of pregnant women about concerning insomnia and depressive symptoms. Healthcare staff should provide preventive and supportive care, when necessary, and should direct pregnant women to providers who can make an early diagnosis and provide treatment.

\section{Funding}

No funding was received for conduction this study.

\section{Conflict of interest}

The authors declare that there is no conflict of interest.

\section{Acknowledgement}

We are express our grateful gratitude to the Professor Behice Erci for assistance with the statistical analysis.

\section{References}

1. Timur S, Şahin NH. Specific quality of life measures for sleep disorders. In: Sahoo S, editor. Can't sleep? Issues of being an insomniac. In Tech; 2012; 39-48.

2. Engin E, Ergün G. Seep and sleep disorders. In: Çam $\mathrm{O}$, Engin E, editors. Mental health and disease nursing care. İstanbul: Istanbul Medical Publishing; 2014; 517-545.

3. Kizilirmak A, Timur S, Kartal B. Insomnia in pregnan- cy and factors related to insomnia. Scientific World Journal. 2012; $1-8$.

4. Özdamar Ö, Yilmaz O, Beyca HH, Muhcu M. Common psychiatric disorders in pregnancy and postpartum period. Zeynep Kamil Medical Bulletin. 2014;45:71-77.

5. Chang JJ, Pien GW, Duntley SP, Macones GA. Sleep deprivation during pregnancy and maternal and fetal outcomes: Is there a relationship? Sleep Med Rev. 2010;14:107114.

6. Hung HM, Ko SH, Chen CH. The association between prenatal sleep quality and obstetric outcome. J Nurs Res. 2014;22:147-153.

7. Román-Gálvez RM, Amezcua-Prieto C, Salcedo-Bellido I, et al. Factors associated with insomnia in pregnancy: A prospective Cohort Study. Eur J Obstet Gynecol Reprod Biol. 2018;221:70-75.

8. Sedov ID, Cameron EE, Madigan S, et al. Sleep quality during pregnancy: A meta-analysis. Sleep Med Rev. 2018;38:168-176 PubMed

9. Rezaei E, Moghadam ZB, Saraylu K. Quality of life in pregnant women with sleep disorder. J Family Reprod Health. 2013;7:87-93.

10. Çoban A, Yanıkkerem UE. Sleep quality and fatigue in pregnant women. Ege Journal of Medicine. 2010;49:87-94. 11. Pinar ŞE, Arslan Ş, Polat K, et al. Examining the association of perceived stress with sleep quality in pregnancy. DEUHFED. 2014;7:171-177.

12. Taşkıran N. Pregnancy and sleep quality. J Turk Soc Obstet Gynecol. 2011;8: 181-187.

13. Çalık KY, Aktaş S. Depression in pregnancy: frequency, risk factors and treatment. Current Approaches to Psychiatry. 2011;3:142-162.

14. Dorheim SK, Bjorvatn B, Eberhard-Gran M. Insomnia and depressive symptoms in late pregnancy: A population-based study. Behav Sleep Med. 2012;10:152-166. 15. Mellor R, Chua SC, Boyce P. Antenatal depression: An artefact of sleep disturbance? Arch Womens Ment Health. 2014;17:291-302.

16. Tsai SY, Lin JW, Kuo LT, et al. Daily sleep and fatigue characteristics in nulliparous women during the third trimester of pregnancy. Sleep. 2012;35:257-62.

17. Çelik F, Köken GN, Yllmazer M. Prevalence of depression during pregnancy and the affecting factors. Anatol J Clin Investig. 2013;7:110-117.

18. Erbil N, Oruç H, Karabulut A. Determination of depression and factors affecting pregnancy. Turkiye Klinikleri J Gynecol Obst. 2009;19:67-74.

19. Eskici L, Akca ASD, Atasoy N, et al. The effects of 
depression and anxiety disorders in pregnant women on obstetric outcomes and newborn. Anatol J Clin Investig. 2012;6:10-6.

20. Rezaei E, Moghadam ZB, Saraylu K. Quality of life in pregnant women with sleep disorder. J Family Reprod Health. 2013;7:87-93.

21. O'Brien LM, Owusu JT, Swanson LM. Habitual snoring and depressive symptoms during pregnancy. $B M C$ Pregnancy Childbirth. 2013;13:2-7.

22. Palagini L, Gemignani A, Banti S, et al. Chronic sleep loss during pregnancy as a determinant of stress: Impact on pregnancy outcome. Sleep Med. 2014;15:853-859.

23. Rezaei E, Moghadam ZB, Hagani H. The effect of sleep health behavioral education on the depression of pregnant women with sleep disorders: A randomized control trial. Iran Red Crescent Med J. 2015;17:1-8.

24. Timur S, Şahin NH. Effects of sleep disturbance on the quality of life of Turkish menopausal women: A population-based study. Maturitas. 2009;64:177-181.

25. Hisli N. A study on the validity of beck depression inventory. Turk Psikol Derg. 1988;6:118-126.

26. Facco FL, Kramer J, Ho KH, et al. Sleep disturbances in pregnancy. Obstet Gynecol. 2010;115:77-83.

27. Ko HS, Shin J, Kim MY, et al. Sleep disturbances in Korean pregnant and postpartum women. J Psychosom Obstet Gynecol. 2012;33:85-90.

28. Cho KO. Sleep Duration and Self-Rated Health are Independently Associated with Physical Activity Level in the Korean Population. Iranian J Publ Health. 2014;43 (5):590-600.

29. Skaer TL. Treatment of insomnia with comorbid mental illness. In: Sahoo S, editor. Can't sleep? Issues of being an insomniac. In Tech; 2012;84-98.
30. Wolynczyk-Gmaj D, RozanskaWaledziak A, Ziem$\mathrm{ka} S$, et al. Insomnia in pregnancy is associated with depressive symptoms and eating at night. J Clin Sleep Med. 2017;13(10):1171-1176.

31. Yang Y, Mao J, Ye Z, et al. Determinants of sleep quality among pregnant women in China: A cross-sectional survey. J Matern Fetal Neonatal Med. 2017;1-6.

32. Şahin KF, Köken G, Coşar E, et al. Obstructive sleep apnea in pregnancy and accompanying diseases. J Turk Soc Obstet Gynecol. 2008;5:22-27.

33. Mourady D, Richa S, Karam R, et al. Associations between quality of life, physical activity, worry, depression and insomnia: a cross-sectional designed study in healthy pregnant women. PLoS One. 2017;12(5):1-15.

34. Ulaşlı SS, Ünlü M. Sleep problems during pregnancy. Updates on Pulmonary Diseases. 2014;2:237-244.

35. Pietikäinen JT, Polo-Kantola P, Pölkki P, et al. Sleeping problems during pregnancy-a risk factor for postnatal depressiveness. Arch Womens Ment Health. 2018;1-11.

36. Skalski M. The diagnosis and treatment of insomnia. In: Sahoo S, editor. Can't sleep? Issues of being an insomniac. In Tech; 2012; 65-80.

37. Yu Y, Li M, Pu L, et al. Sleep was associated with depression and anxiety status during pregnancy: a prospective longitudinal study. Arch Womens Ment Health. 2017;20:695-701.

32.

38. Tsai SY, Kuo LT, Lee CN, et al. Reduced sleep duration and daytime naps in pregnant women in Taiwan. Nurs Res. 2013;62:99-105.

39. Kaya B. Depression: socio-economic and cultural perspectives. J Clin Psy. 2007;10:11-20. 